\title{
Structure-Dependent Mechanical Properties of ALD-Grown Nanocrystalline $\mathrm{BiFeO}_{3}$ Multiferroics
}

\author{
Anna Majtyka, ${ }^{1}$ Anna Nowak, ${ }^{2}$ Benoît Marchand, ${ }^{3}$ Dariusz Chrobak, ${ }^{4}$ Mikko Ritala, ${ }^{5}$ \\ Jyrki Räisänen, ${ }^{3}$ and Roman Nowak ${ }^{1}$ \\ ${ }^{1}$ Nordic Hysitron Laboratory, Department of Materials Science and Engineering, Aalto University, 00076 Espoo, Finland \\ ${ }^{2}$ A. Chelkowski Institute of Physics, University of Silesia, Uniwersytecka 4, 40-007 Katowice, Poland \\ ${ }^{3}$ Department of Physics, University of Helsinki, P.O. Box 43, 00014 Helsinki, Finland \\ ${ }^{4}$ Institute of Material Sciences, University of Silesia, 75 Pulku Piechoty 1A, 41-500 Chorzow, Poland \\ ${ }^{5}$ Department of Chemistry, Laboratory of Inorganic Chemistry, University of Helsinki, P.O. Box 55, 00014 Helsinki, Finland
}

Correspondence should be addressed to Roman Nowak; nowak.roman5@gmail.com

Received 17 February 2016; Accepted 5 April 2016

Academic Editor: Jeffrey Elam

Copyright (C) 2016 Anna Majtyka et al. This is an open access article distributed under the Creative Commons Attribution License, which permits unrestricted use, distribution, and reproduction in any medium, provided the original work is properly cited.

\begin{abstract}
The present paper pertains to mechanical properties and structure of nanocrystalline multiferroic $\mathrm{BeFiO}_{3}(\mathrm{BFO})$ thin films, grown by atomic layer deposition (ALD) on the $\mathrm{Si} / \mathrm{SiO}_{2} / \mathrm{Pt}$ substrate. The usage of sharp-tip-nanoindentation and multiple techniques of structure examination, namely, grazing incidence X-ray diffraction, X-ray photoelectron spectroscopy, scanning electron microscopy, and energy dispersive X-ray spectrometry, enabled us to detect changes in elastic properties ( $95 \mathrm{GPa} \leq E \leq 118 \mathrm{GPa})$ and hardness ( $4.50 \mathrm{GPa} \leq H \leq 7.96 \mathrm{GPa}$ ) of $\mathrm{BFO}$ after stages of annealing and observe their relation to the material's structural evolution. Our experiments point towards an increase in structural homogeneity of the samples annealed for a longer time. To our best knowledge, the present report constitutes the first disclosure of nanoindentation mechanical characteristics of ALD-fabricated $\mathrm{BeFiO}_{3}$, providing a new insight into the phenomena that accompany structure formation and development of nanocrystalline multiferroics. We believe that our systematic characterization of the BFO layers carried out at consecutive stages of their deposition provides pertinent information which is needed to control and optimize its ALD fabrication.
\end{abstract}

\section{Introduction}

The $\mathrm{BiFeO}_{3}$ compounds attract currently a significant interest due to their prominent multiferroicity that concerns their superior ferroelectric and (anti-)ferromagnetic properties. These fascinating materials give us a possibility to control ferroelectricity and ferromagnetism by means of a magnetic or electric field $[1,2]$. Their magnetoelectric coupling and strong electric polarization raises the prospect of using these for data storage, magnetic detectors, and tunnel-junction devices or as essential spintronics materials [2-4].

There are numerous methods for production of BFO thin films, such as pulsed laser deposition (PLD) [5], metalorganic chemical vapor deposition (MOCVD) [6], radio frequency magnetron sputtering [7], or sol-gel solution deposition [8], to name merely a few. As is well known, the synthesis procedure has a pronounced effect on physical properties of different materials [9]; therefore establishing of a manufacturing process is very important. The present paper pertains to BFO thin films obtained via the atomic layer deposition (ALD) technique being nowadays widely appreciated due to, for instance, its capability to deposit various geometries with thickness control [10] or produce multiferroic materials with satisfying dielectric properties [11]. Thus, we employed the ALD procedure to obtain nanocrystalline BFO thin films which underwent nanomechanical probing, allowing us to investigate the mechanical properties and characterize the structure of our material, which is essential for technological applications of $\mathrm{BFO}[12,13]$.

Although the electric and magnetic properties of $\mathrm{BiFeO}_{3}$ compound are already well recognized [11], there is an increasing interest in mechanical properties of multiferroic 
materials of industrial importance, especially in these produced in a nanostructured form [14]. Since the nanoindentation testing already proved to be a powerful tool to investigate this kind of materials $[7,8]$, we employed the surface-deformation measurements to reveal the relationship between mechanical behavior of BFO and its structure. Such an approach enabled one to conclude on elastic and plastic responses, stress-induced phase-transitions $[15,16]$, and dislocation-based mechanisms of nanodeformation in semiconductors and metals $[15,17]$, while the present paper demonstrates mechanical and structural changes in BFO produced via ALD, when various annealing times are used. Young modulus, hardness, and grain size of the material in question are determined. To our best knowledge, this study for the first time deals with the evaluation of multiferroic material properties during consecutive stages of its annealing process, until the crystalline BFO material is received.

\section{Experimental}

2.1. Material Synthesis and Preparation of the Samples. $\mathrm{BiFeO}_{3}$ thin films were prepared by the ALD method using a process composed of two deposition stages. Firstly, deposition of initial $\mathrm{Bi}-\mathrm{O}$ and $\mathrm{Fe}-\mathrm{O}$ layers on an $\mathrm{Si} / \mathrm{SiO} \mathrm{S}_{2} / \mathrm{Pt}$ substrate was carried out at $413 \mathrm{~K}$, using bismuth(III) 2,3-dimethyl2-butoxide and iron(III) tert-butoxide as metal precursors. We applied an exact number of growth-cycles (200 cycles of iron oxide and 180 of bismuth oxide) followed by exposure of the material to water vapor in order to oxidize the deposited layers. This operation was repeated 15 times to achieve a satisfactory layered structure of $\mathrm{Bi}-\mathrm{O} / \mathrm{Fe}-\mathrm{O}$ oxides. Secondly, the fabricated laminar "semiproducts" underwent annealing in nitrogen atmosphere at $823 \mathrm{~K}$. The nonannealed samples were also secured for a further examination, for the sake of comparison. The duration of the applied thermal treatment varied for different sets of specimens denoted finally as S5, S-30, and S-60 for the films annealed for 5, 30, and 60 minutes, respectively. Our multilayers were $500 \mathrm{~nm}$ thick, similar to those produced by Jalkanen et al., who considered only the S-60 material as the one with a well-developed $\mathrm{BiFeO}_{3}$ crystalline structure (details in [11]).

2.2. Structural Analysis and Mechanical Testing. The crystalline structure of the ALD-prepared BFO material was determined by means of X-ray diffraction (PANalytical X'pert Pro diffractometer with $\mathrm{CuK}_{\alpha} \mathrm{X}$-ray source, $\lambda=1.5405 \AA$ ) that enabled us to conclude on the grain size of the formed structure. The complementary evaluation of the obtained microstructure and the relative content of different elements were carried out using a Scanning Electron Microscope (SEM) equipped with an Energy Dispersive X-ray Spectrometer (EDS) within a Hitachi S-4800 (Oxford INCA 350 spectrometer) system. The quantitative analysis of composition of the obtained materials was made by means of X-ray Photoelectrons Spectroscopy (XPS) with monochromatized $\mathrm{Al} \mathrm{K} \alpha$ radiation. In order to reveal the depth-distribution of investigated elements, Elastic Recoil Detection Analysis (ERDA) was employed to finally determine the structure of the ALD-produced BFO.
As far as the mechanical properties are concerned, we examined the prepared BFO materials using well-established nanoindentation experiments carried out with Hysitron Triboindenter TI 950 armed with a sharp diamond tip (Berkovich-type, radius of $R=100 \mathrm{~nm}$ ) and alternatively a spherical one (radius of $R=2 \mu \mathrm{m}$ ). We intended to reveal changes in the mechanical response of the BFO multiferroic prior to and after the annealing process, in order to obtain a pertinent characteristic for the deposited material. Moreover, the nanoindentation results constitute a convenient supportive feedback for the control of the applied ALD fabrication process. We determined the essential mechanical parameters, namely, the elastic modulus $E$ and hardness $H$ of our films, according to the Oliver and Pharr method [20] with the central relationships that reads

$$
\begin{aligned}
H & =\frac{P}{A}, \\
\frac{1-v^{2}}{E} & =\frac{2 \beta}{S \sqrt{\pi}} \sqrt{A}-\frac{1-v_{i}^{2}}{E_{i}},
\end{aligned}
$$

where $P$ is the indentation load, $A$ is projected contact area, $\beta$ is constant dependent on the tip geometry, $S$ is unloading stiffness, $v$ is film's Poisson's ratio, $v_{i}$ is Poisson's ratio of a diamond, and $E_{i}$ is tip (diamond) elastic modulus, respectively.

Our nanoindentation experiments were carried out with a maximum load $P_{\max }$ ranging from 200 to $1000 \mu \mathrm{N}$ in order to satisfy the commonly accepted practice that recommends refraining from exceeding the maximum indentation depth more than $10 \%$ of the investigated film thickness [21]. The selection of a smooth surface area, free of defects, and therefore suitable for nanoindentation measurements, was accomplished using Scanning Probe Microscopy (SPM) based on surface profiling by the tip being in contact with a film, which is available in our TI 950 instrument.

\section{Results and Discussion}

The structure of our ALD-grown materials was determined by grazing incidence X-ray diffraction. The examinations revealed a predominantly amorphous structure in the specimens that did not undergo the annealing process, while the others (namely, those heated for $5 \mathrm{~min}$ and longer) crystallized as a stable $\mathrm{BiFeO}_{3}$-phase (Figure 1). A hexagonal $R 3 c$ (161) BFO structure with lattice constants of $a=b=$ 5.5876(3) $\AA$ and $c=13.867$ (1) $\AA$ was detected. The two highest diffraction peaks recorded for the sample annealed for 5 minutes are broader and lower (Figure 1) than those observed for the film annealed for 60 minutes, which confirms a typical behavior of annealed polycrystals. The higher intensity suggests a higher content of the component generating the peak, in the present case, the increased amount of the BFO phase. The diffraction peaks at Bragg angle $\Theta=39.9^{\circ}$ and $\Theta=46.3^{\circ}$ correspond to Pt (111) and Pt (200) plane since our BFO samples were heterogeneously ALD-grown on a $\mathrm{Si} / \mathrm{SiO}_{2} / \mathrm{Pt}$ substrate. Interestingly, the spectrum recorded for $\mathrm{S}-60$ reveals a smaller contribution of the $\mathrm{Pt}$ phase into the deposited $\mathrm{BiFeO}_{3}$ film. 


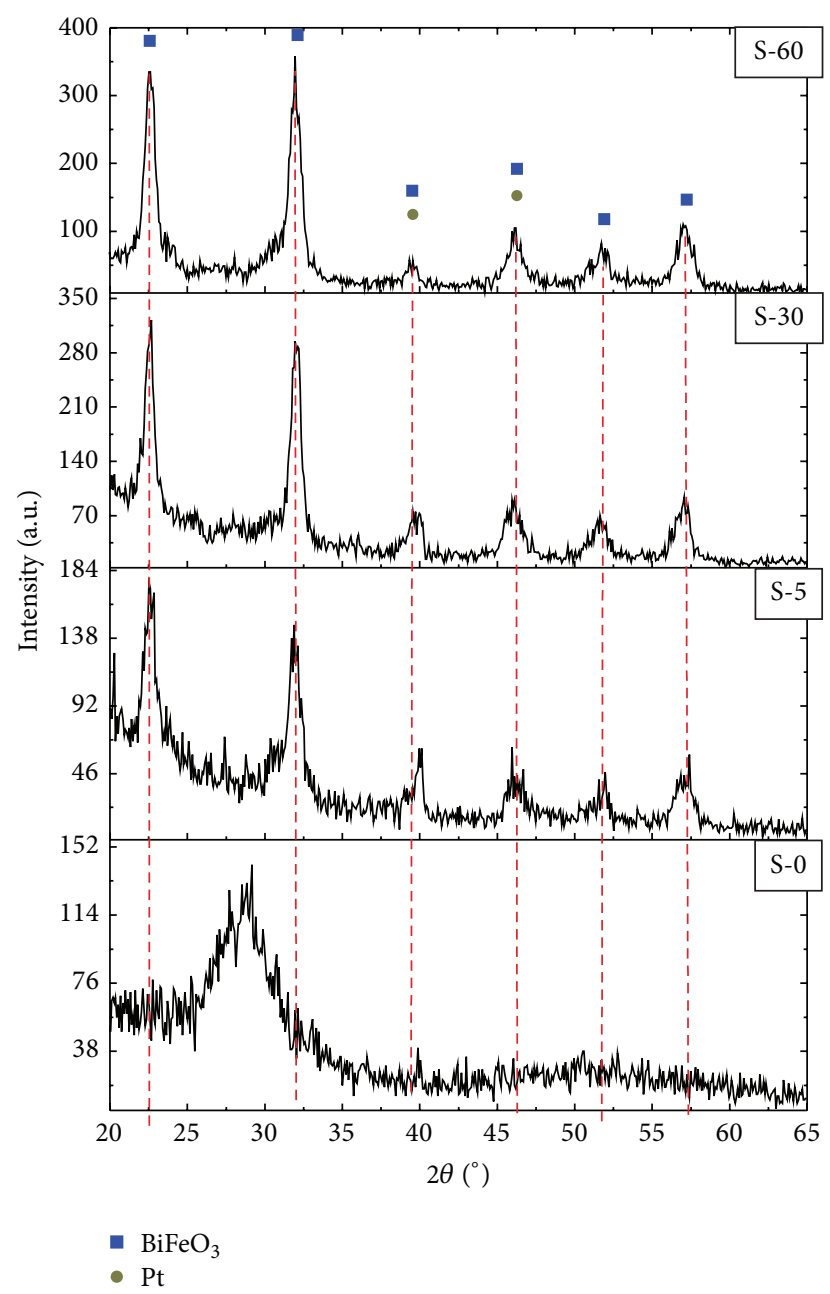

FIgURE 1: XRD spectra recorded for $\mathrm{BiFeO}_{3}$ thin films deposited by the ALD method on the $\mathrm{Si} / \mathrm{SiO}_{2} / \mathrm{Pt}$ substrate and subsequently annealed for $5,15,30$, or 60 minutes (the samples S- 0, S-5, S-30, and S-60 according to our notation).

Moreover, the grain size of the crystallite was determined using Scherrer's equation [22]:

$$
D=\frac{k \lambda}{\beta_{w} \cos \theta},
$$

where $D$ is the crystallite size, $k$ is a dimensionless shape factor, $\lambda$ is the $\mathrm{X}$-ray wavelength, $\beta_{w}$ is the line width at half maximum intensity of the peak, and $\theta$ is Bragg angle. Thus, the estimated grain size equalled $5.58,10.06$, and $8.66 \mathrm{~nm}$ for the annealed S-5, S-30, and S-60 samples, respectively.

The morphology of the deposited material was carefully examined by means of SEM-technique coupled with EDS analysis. The obtained micrographs (Figure 2) proved that the surface of the obtained specimens is homogeneous and smooth (see Figure 2). They also revealed a commonly expected increase of the grain size that occurs during the annealing process [7]. Interestingly, beyond the annealingtime-threshold of 30 minutes, we did not observe further changes in the surface morphology (Figure 2), and the
TABLE 1: The Bi/Fe ratio concluded from EDS measurements carried out for $\mathrm{BiFeO}_{3}$ thin films.

\begin{tabular}{ll}
\hline Sample & Bi/Fe ratio \\
\hline Nonannealed & $1.07 \pm 0.02$ \\
5 min & $1.03 \pm 0.02$ \\
$30 \mathrm{~min}$ & $1.04 \pm 0.02$ \\
$60 \mathrm{~min}$ & $0.97 \pm 0.02$ \\
\hline
\end{tabular}

structure seemed already well stabilized. This conclusion is supported by the results of the EDS measurements which enabled us to estimate the $\mathrm{Bi} / \mathrm{Fe}$ content ratio of the examined samples (refer to Table 1). Thus, the stoichiometry proper for $\mathrm{BiFeO}_{3}$ was confirmed exclusively in the case of the S-60 material.

The results of the XPS examinations revealed a principal core level of $\mathrm{Bi}, \mathrm{Fe}$, and $\mathrm{O}$ ion content and confirmed the presence of $\mathrm{C}, \mathrm{F}$, and $\mathrm{Si}$ in the samples structure. The spectra were corrected for the background signal using the iterated Shirley algorithm, while the band levels were adjusted using Gaussian and Lorentzian lines. The presence of characteristic spectral lines for Fe2p core levels (Figure 3(a)) proved that $\mathrm{FeO}$ with binding energy of $E_{b}=709.9 \mathrm{eV}$ and $\mathrm{Fe}_{2} \mathrm{O}_{3}\left(E_{b}=\right.$ $710.8 \mathrm{eV}$ ) are included among the main components of our film. The same applies to the Bi4f (Figure 3(b)) core level and consequently to the $\mathrm{Bi}_{2} \mathrm{O}_{3}$ phase $\left(E_{b}=158.95 \mathrm{eV}\right)$. The satellite peak for $\mathrm{Fe}^{3+}$ (Figure 3(a)) located at $719 \mathrm{eV}$ suggests a partial oxidation of our samples, which is in accordance with the observation by Li et al. [23]. Despite considerable difficulties in detection of the contribution from the $\mathrm{Fe}$ and Bi oxide from the O1s core level (Figure 3(c)), we successfully determined the binding energy of $529.85 \mathrm{eV}$ for both $\mathrm{Bi}$ and Fe oxides using the NIST database. Furthermore, the $\mathrm{SiO}_{2}$ signal from the substrate that we used for BFO deposition was also registered. Thus, the investigation of chemical state of our material confirmed the presence of pure BFO phase.

For the sake of clarity of our paper, we carried out the indentation examination for the nonannealed (S-0) and longannealed (S-60) BFO thin films only. Our SPM probing of the surface of the nonannealed (Figure 4(a)) and annealed (Figure 4(b)) specimens revealed a significant difference in the surface morphology. The nanoindentation measurements were carried out on a carefully selected area of the samples with a similar roughness. It was possible due to the nanometer-scale contact area induced by our indenter. Thus, our nanoindentation results can be considered as virtually unaffected by the displayed surface features (Figures 4(a)$4(\mathrm{~b}))$.

The micrographs in Figures 4(a) and 4(b) disclose a difference in the surface roughness for the nonannealed and annealed specimens. However, the marked consistency and repeatability of the obtained results (refer to Figure 4(c)) convince us that the noted discrepancies do not affect the results of mechanical testing. Consequently, we reported a considerable change in mechanical properties of the BFO specimen when annealed for a longer time (compare black and red curves in Figure 4(c)). Despite the character of the $P$ - $h$ curves being of a similar kind, the material after 


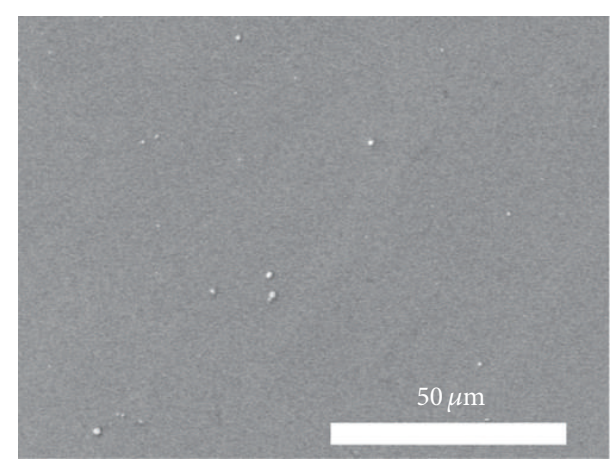

(a) S-0

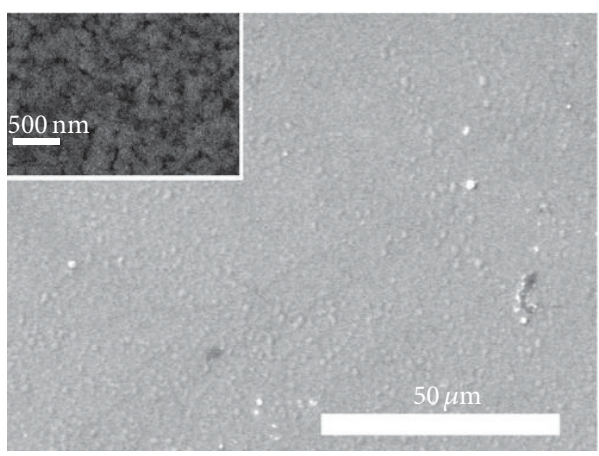

(c) S-30

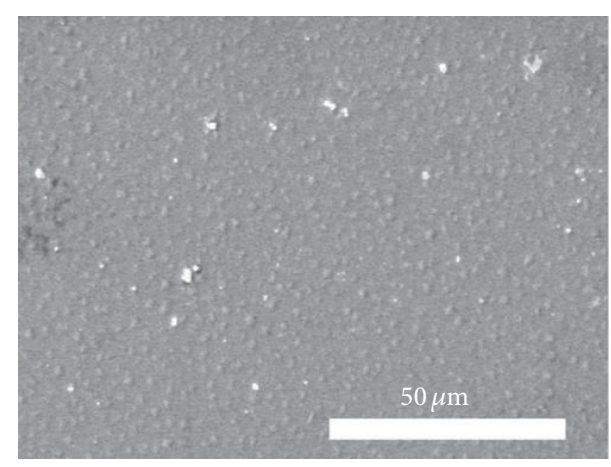

(b) S-5

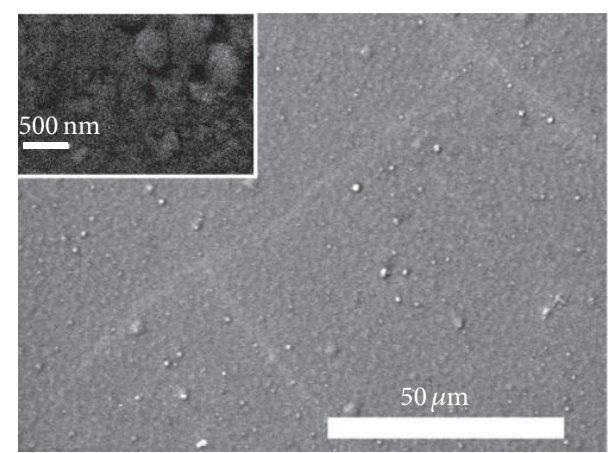

(d) S-60

Figure 2: SEM micrographs of BFO films after annealing at $T=300 \mathrm{~K}$ for $0,5,30$, and $60 \mathrm{~min}$ (the samples denoted as S-0, S-5, S-30, and $\mathrm{S}-60$ ). The insets ( $c$ and d) reveal structural aspects in a greater detail, which disclose a difference between the S-60 sample (d) which contains $\mathrm{BiFeO}_{3}$ structure (refer to Figure 1) and the other materials (a, b, c).

TABLE 2: Elastic modulus and hardness of S-0 and S-60 BFO thin films obtained from different deposition methods.

\begin{tabular}{lcc}
\hline Method & $E(\mathrm{GPa})$ & $H(\mathrm{GPa})$ \\
\hline Atomic layer deposition (ALD) & $111.11-118.31$ & $7.50-7.96$ \\
Radio frequency magnetron sputtering [7] & $131.4-170.8$ & $6.8-10.6$ \\
Sol-gel [8] & $26-51$ & $2.8-3.8$ \\
Pulsed laser deposition [18] & 100 & - \\
First-principles calculation [19] & G-AFM - 142.19 & - \\
\hline
\end{tabular}

the thermal treatment (S-60) becomes markedly harder (a significantly lower indentation depth $h$ ) than the ALD-grown one $(\mathrm{S}-0)$, which runs contrary to common expectation (Figure 4(c)). Indeed, polycrystalline materials (especially metals) usually turn softer after annealing [7], which contrasts with the discovered BFO behavior. All of these bring us to suspect that the observed hardening is due to considerable structural changes which occur during annealing.

The deformation of the amorphous material (S-0, black curve) and the one crystallized during annealing (see Figure 4) of the BFO films (S-60, red curves) performed within a load range from $P_{\max }=200 \mu \mathrm{N}$ to $1000 \mu \mathrm{N}$ does not resemble either the results obtained for $\mathrm{GaN}$ [15] and GaAs [16] crystals, in which nanodeformation depends on the transition between crystalline structures, accompanied by a dislocation activity.
Our analysis of the nanoindentation $P-h$ data is supported by classical hardness and elastic modulus measurements that confirm that the heat treatment resulted in increase of these two. Furthermore, the results of our nanomechanical testing were compared with those obtained by other authors for different deposition methods and various kinds of measurements, or they were predicted theoretically (Table 2). Thus, we contend that the nanoindentation probing and the recorded mechanical characteristics serve to be a convenient indicator of the process that undergoes between $\mathrm{Bi}-\mathrm{O}$ and $\mathrm{Fe}$ $\mathrm{O}$ layers with increasing time of heat treatment.

Our results reveal a decrease in hardness and elastic moduli with an increasing load, which reflects the wellknown "size effect" [24]. The corresponding Young modulus values (hardness) for BFO material are $118.31 \mathrm{GPa}(7.96 \mathrm{GPa})$, $114.43 \mathrm{GPa}(7.80 \mathrm{GPa})$, and $111.11 \mathrm{GPa}(7.50 \mathrm{GPa})$ for loads 


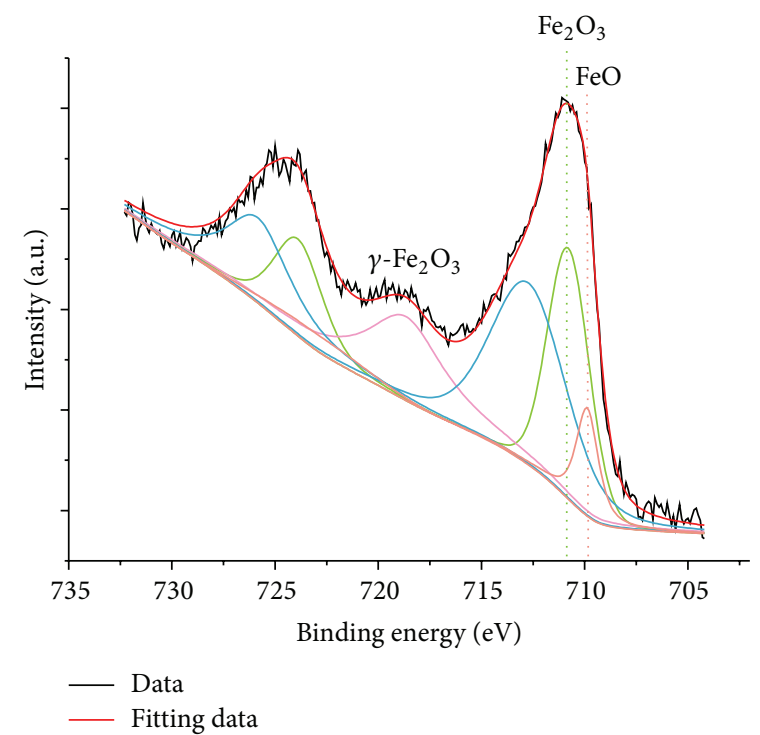

(a)

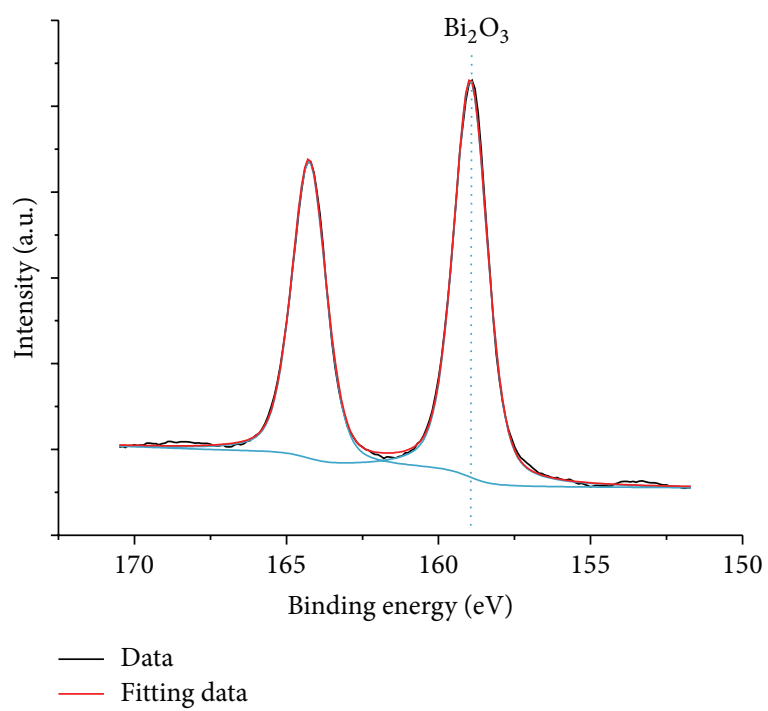

(b)

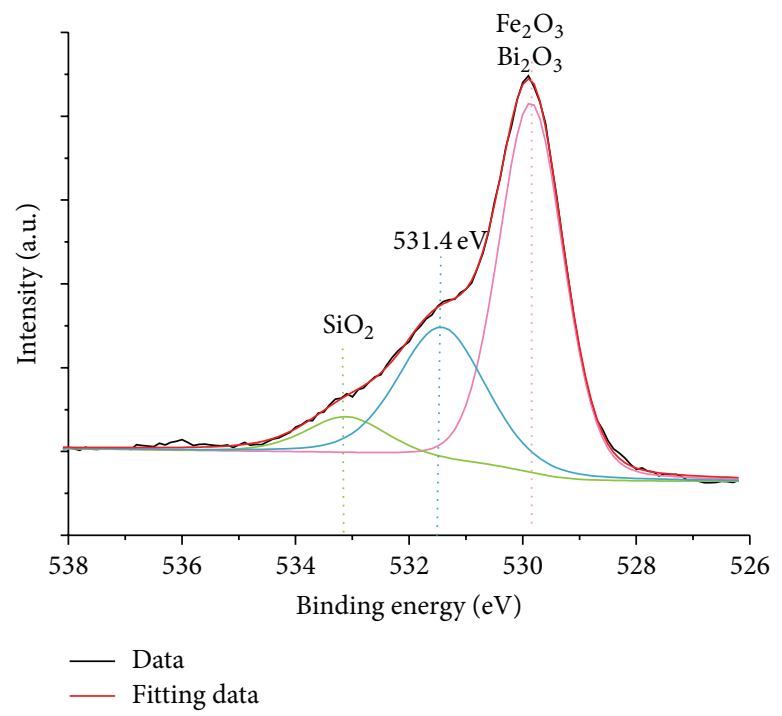

(c)

FIgURE 3: XPS spectra obtained for a $500 \mathrm{~nm} \mathrm{BiFeO}_{3}$ film (S-60) grown by the ALD technique. One can readily recognize the spectral components for Fe2p (a), Bi4f (b), and also O1s lines (c).

(depths) of $200 \mu \mathrm{N}(25 \mathrm{~nm}), 600 \mu \mathrm{N}(52 \mathrm{~nm})$, and $1000 \mu \mathrm{N}$ $(76 \mathrm{~nm})$, respectively.

One should note that hardness of the nonannealed sample $(H=4.5 \mathrm{GPa})$ is almost two times lower than that of the one which underwent the annealing procedure, and its elastic modulus is also lower ( $95 \mathrm{GPa}$ ).

\section{Conclusions}

The present work focused on the relationship between mechanical characteristics and structure of industrially important multiferroic $\mathrm{BiFeO}_{3}$ films ALD grown on an $\mathrm{Si} / \mathrm{SiO}_{2} / \mathrm{Pt}$ substrate and subsequently variously annealed. This way, we revealed a dependence of the common mechanical parameters on the stage of crystallization of BFO films and finally formation of the $\mathrm{BiFeO}_{3}$ structure. Thus, we contend that nanoindentation constitutes a simple and very convenient method of testing multiferroic films with respect to formation of a properly developed structure of the material.

In a greater detail, a number of measurements were carried out to determine both structure and mechanical properties of the BFO thin film that underwent a post-ALD thermal treatment (annealing) at $823 \mathrm{~K}$ which lasted for 5, 30 , and 60 minutes. The as-deposited samples (S-0) were also examined as a reference material. Thus, the structures of the samples labelled as S-0, S-5, S-30, and S-60 were extensively investigated by means of XRD, XPS, SEM, and EDS techniques.

Since we found that our ALD-grown BFO layers require at least 60 minutes of treatment to crystallize in the intact 


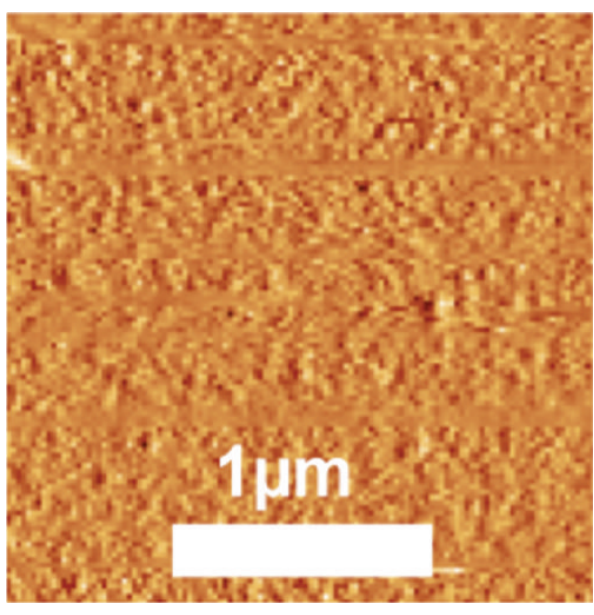

(a)

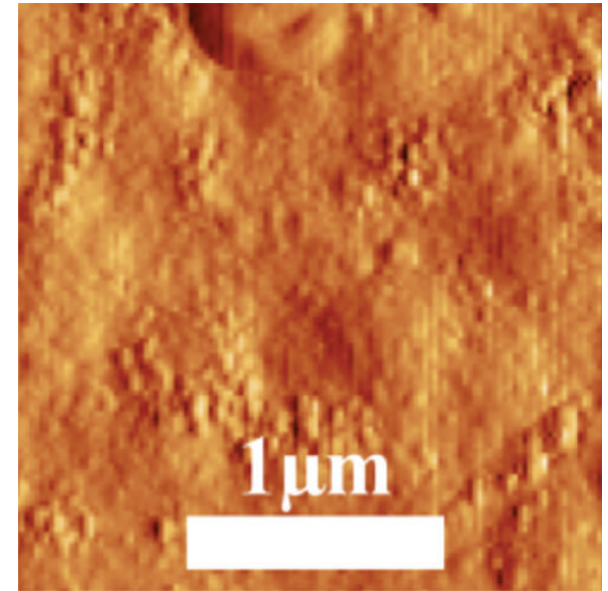

(b)

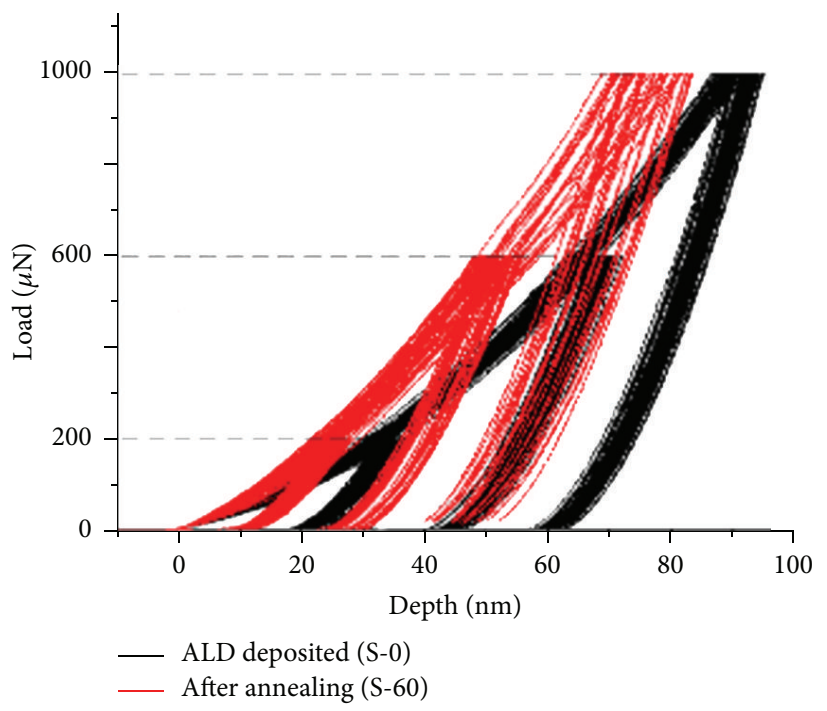

(c)

Figure 4: The surface morphology of the BFO samples S-0 (a) and S-60 (b) intended for nanomechanical testing and the results of nanoindentation examinations (c). The SPM micrographs of selected area of the S- 0 and S-60 samples reveal features characteristic for an ALD-grown (a) and thoroughly annealed $(t=60 \mathrm{~min}, T=300 \mathrm{~K})$ material (b). The nanoindentation load-depth $P$ - $h$ curves are recorded for various maximum loads $P_{\max }$ of 200, 400, and $1000 \mu \mathrm{N}$, for both S-0 (black) and S-60 (red) thin films (c).

$\mathrm{BiFeO}_{3}$ structure, the mechanical testing was limited to as-deposited (S-0) and final (S-60) state proving that the nanoindentation testing is a perfect experimental method to provide us the information about the stage of formation of a multiferroic material.

Furthermore, our research provides (to the best of our knowledge for the first time) nanoindentation mechanical characteristics of BFO multiferroics grown by means of the ALD process. Hardness obtained under nominal loads of 200,600 , and $1000 \mu \mathrm{N}$ was equal to $7.96,7.80$, and $7.50 \mathrm{GPa}$, respectively (please note that hardness is a loaddependent material property that reflects plastic properties of the material) [25]. In contrast hardness of the as-deposited film amounted to $4.5 \mathrm{GPa}$. As for elastic characteristics, the level elastic moduli of a properly crystallized $\mathrm{BiFeO}_{3}$ equalled $118.31,114.43$, and $111.11 \mathrm{GPa}$ under various loads, while that of the as-grown material (S-0) approached $\sim 95 \mathrm{GPa}$. The almost negligible load/depth-dependence of $E r$ - and $H$-parameter of our sample proved a homogeneity of the material and correctness of our measurements.

\section{Competing Interests}

The authors declare that they have no competing interests.

\section{Acknowledgments}

The authors thank the Academy of Finland for the support through the Research Consortium PROPER within the Finnish Research Program for PROGRAMABLE MATERIALS (OMA) and the Finnish Centre of Excellence in Atomic Layer Deposition. Dariusz Chrobak acknowledges support 
from the National Science Center of Poland under Grant number DEC-2012/05/B/ST-8/02945.

\section{References}

[1] M. Fiebig, Th. Lottermoser, D. Fröhlich, A. V. Goltsev, and R. V. Pisarev, "Observation of coupled magnetic and electric domains," Nature, vol. 419, no. 6909, pp. 818-820, 2002.

[2] Y. Chu, L. W. Martin, M. B. Holcomb et al., "Electric-field control of local ferromagnetism using a magnetoelectric multiferroic," Nature Materials, vol. 7, no. 6, pp. 478-482, 2008.

[3] S. Boyn, S. Girod, V. Garcia et al., "High-performance ferroelectric memory based on fully patterned tunnel junctions," Applied Physics Letters, vol. 104, no. 5, Article ID 052909, 2014.

[4] N. A. Spaldin and M. Fiebig, "The renaissance of magnetoelectric multiferroics," Science, vol. 309, no. 5733, pp. 391-392, 2005.

[5] K.-Y. Yun, D. Ricinschi, M. Noda, M. Okuyama, and S. Nasu, "Ferroelectric and magnetic properties of multiferroic $\mathrm{BiFeO}_{3}$ thin films prepared by pulsed laser deposition," Journal of the Korean Physical Society, vol. 46, no. 1, pp. 281-284, 2005.

[6] S. Y. Yang, F. Zavaliche, L. Mohaddes-Ardabili et al., "Metalorganic chemical vapor deposition of lead-free ferroelectric $\mathrm{BiFeO}_{3}$ films for memory applications," Applied Physics Letters, vol. 87, Article ID 102903, 2005.

[7] S.-R. Jian, H. W. Chang, Y. C. Tseng, P. H. Chen, and J. Y. Juang, "Structural and nanomechanical properties of $\mathrm{BiFeO}_{3}$ thin films deposited by radio frequency magnetron sputtering," Nanoscale Research Letters, vol. 8, article 297, 2013.

[8] P. Sen, A. Dey, A. K. Mukhopadhyay, S. K. Bandyopadhyay, and A. K. Himanshu, "Nanoindentation behaviour of nano $\mathrm{BiFeO}_{3}$," Ceramics International, vol. 38, no. 2, pp. 1347-1352, 2012.

[9] A. Rousset, "Specific electrical, magnetic, and magneto-optical properties of materials manufactured by 'chimie douce," Solid State Ionics, vol. 84, no. 3-4, pp. 293-301, 1996.

[10] V. Miikkulainen, M. Ritala, M. Zeskelä, and R. L. Puurunen, "Crystallinity of inorganic films grown by atomic layer deposition: overview and general trends," Journal of Applied Physics, vol. 113, no. 2, Article ID 021301, 2013.

[11] P. Jalkanen, V. Tuboltsev, B. Marchand et al., "Magnetic properties of polycrystalline bismuth ferrite thin films grown by atomic layer deposition," The Journal of Physical Chemistry Letters, vol. 5, no. 24, pp. 4319-4323, 2014.

[12] G. Catalan and J. F. Scott, "Physics and applications of bismuth ferrite," Advanced Materials, vol. 21, no. 24, pp. 2463-2485, 2009.

[13] A. R. Akbashev, G. Chen, and J. E. Spanier, "A facile route for producing single-crystalline epitaxial perovskite oxide thin films," Nano Letters, vol. 14, no. 1, pp. 44-49, 2014.

[14] N. Domingo, J. Narvaez, M. Alexe, and G. Catalan, "Local properties of the surface layer(s) of $\mathrm{BiFeO}_{3}$ single crystals," Journal of Applied Physics, vol. 113, Article ID 187220, 2013.

[15] D. Chrobak, K.-H. Kim, K. J. Kurzydłowski, and R. Nowak, "Nanoindentation experiments with different loading rate distinguish the mechanism of incipient plasticity," Applied Physics Letters, vol. 103, no. 7, Article ID 072101, 2013.

[16] R. Nowak, D. Chrobak, S. Nagao et al., "An electric current spike linked to nanoscale plasticity," Nature Nanotechnology, vol. 4, no. 5, pp. 287-291, 2009.

[17] C. L. Kelchner, S. J. Plimpton, and J. C. Hamilton, "Dislocation nucleation and defect structure during surface indentation," Physical Review B, vol. 58, no. 17, pp. 11085-11088, 1998.
[18] F. Bai, J. Wang, M. Wuttig et al., "Destruction of spin cycloid in $(111)_{c}$-oriented $\mathrm{BiFeO}_{3}$ thin films by epitiaxial constraint: enhanced polarization and release of latent magnetization," Applied Physics Letters, vol. 86, Article ID 032511, 2005.

[19] H. Dong, C. Chen, S. Wang, W. Duan, and J. Li, "Elastic properties of tetragonal $\mathrm{BiFeO}_{3}$ from first-principles calculations," Applied Physics Letters, vol. 102, no. 18, Article ID 182905, 2013.

[20] W. C. Oliver and G. M. Pharr, "Improved technique for determining hardness and elastic modulus using load and displacement sensing indentation experiments," Journal of Materials Research, vol. 7, no. 6, pp. 1564-1580, 1992.

[21] T. Y. Tsui and G. M. Pharr, "Substrate effects on nanoindentation mechanical property measurement of soft films on hard substrates," Journal of Materials Research, vol. 14, no. 1, pp. 292-301, 1999.

[22] P. Scherrer, "Bestimmung der Grösse und der inneren Struktur von Kolloidteilchen mittels Röntgenstrahlen," Nachrichten von der Gesellschaft der Wissenschaften zu Göttingen, vol. 26, pp. 98100, 1918.

[23] P. Li, E. Y. Jiang, and H. L. Bai, "Fabrication of ultrathin epitaxial $\gamma-\mathrm{Fe}_{2} \mathrm{O}_{3}$ films by reactive sputtering," Journal of Physics D: Applied Physics, vol. 44, no. 7, Article ID 075003, pp. 1-5, 2011.

[24] W. D. Nix and H. Gao, "Indentation size effects in crystalline materials: a law for strain gradient plasticity, Journal of the Mechanics and Physics of Solids, vol. 46, no. 3, pp. 411-425, 1998.

[25] R. Nowak, F. Yoshida, D. Chrobak, K. J. Kurzydłowski, T. Takagi, and T. Sasaki, "Nanoindentation examination of crystalline solid surface," in Encyclopedia of Nanoscience and Nanotechnology, S. H. Nalwa, Ed., pp. 313-374, American Association, Philadelphia, Pa, USA, 2011. 

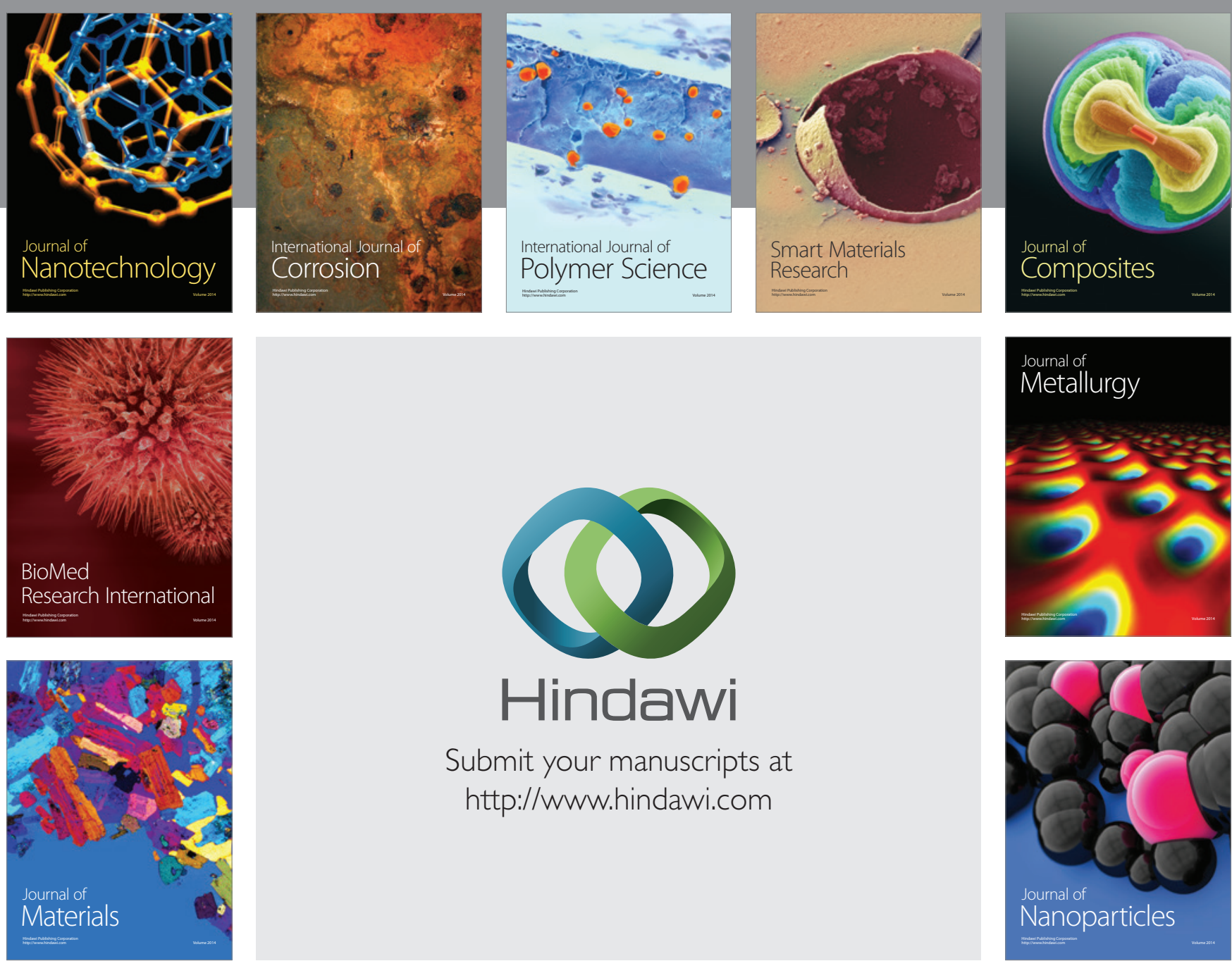

\section{Hindawi}

Submit your manuscripts at

http://www.hindawi.com

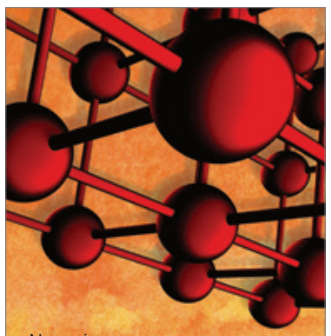

Materials Science and Engineering
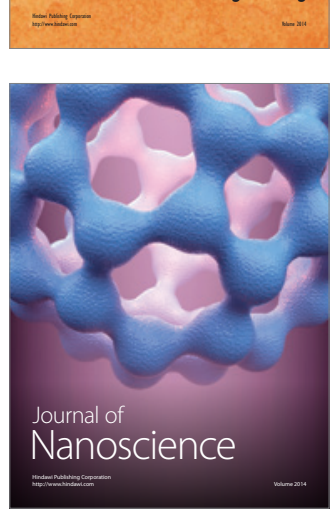
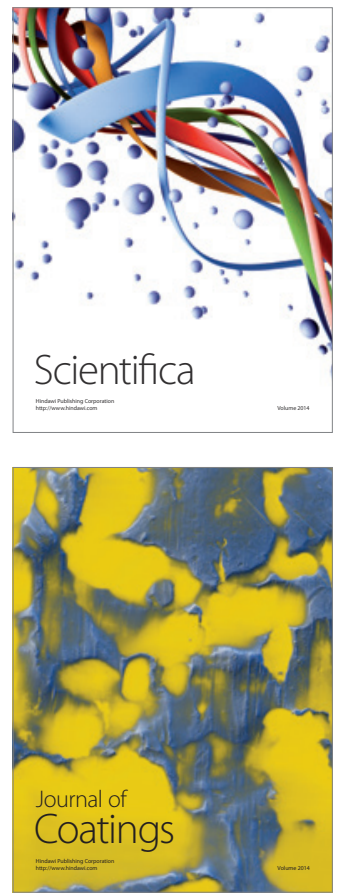
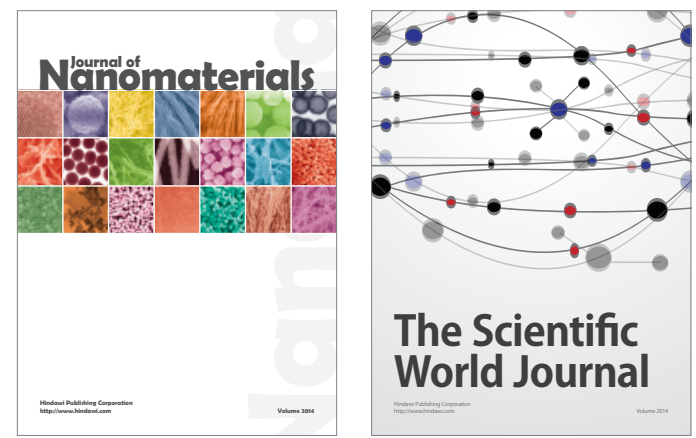

The Scientific World Journal
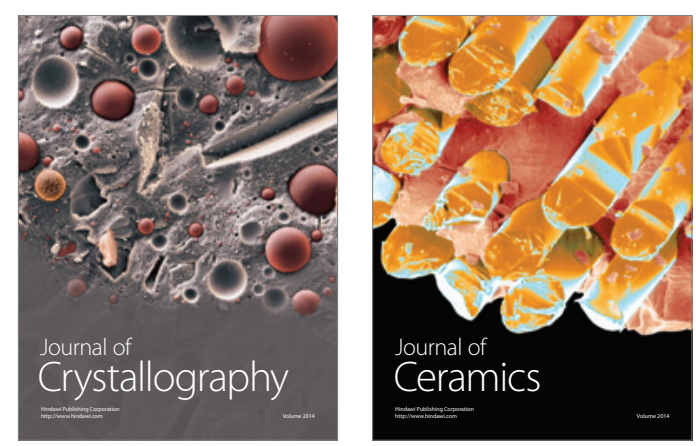
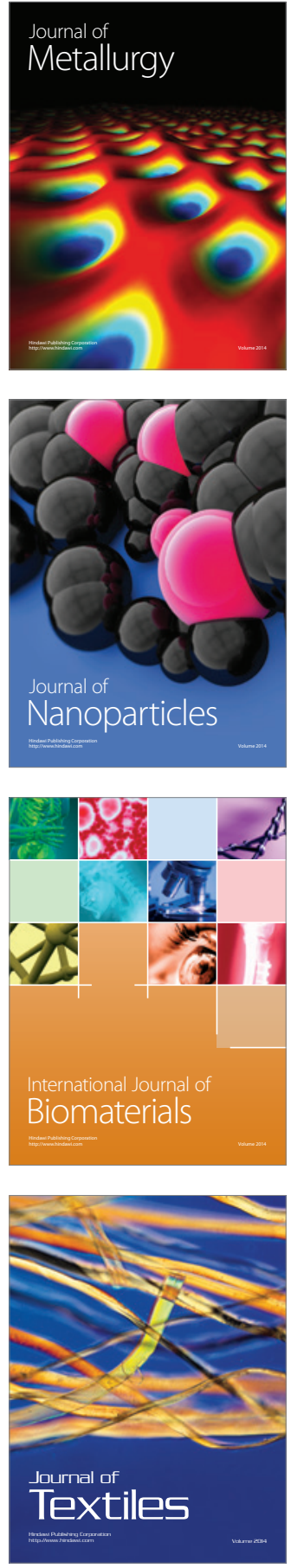